\title{
La Historia del Departamento de Obstetricia y Ginecología - Facultad de Medicina - Universidad Nacional de Colombia
}

\author{
Heliodoro Ñáñez Burbano*
}

La historia del Departamento de Ginecología y Obstetricia está intrínsecamente ligada a la de la Facultad de Medicina de la Universidad Nacional y a la del Instituto Materno Infantil.

La Escuela de Medicina de la Universidad Nacional inició labores en febrero de 1968; cinco años más tarde se estableció en la Clínica Obstétrica e Infantil, en el Hospital San Juan de Dios, situado para ese entonces en la carrera 9a. con calle 11 (antes del Hospital funcionaba en la carrera 6a., detrás de la Catedral), dirigida por el Dr. Leoncio Barreto y José María Buendía.

En el año de 1912 el Servicio de Maternidad estaba dirigido por el Profesor Miguel Rueda Acosta y sus jefes de clínica eran los doctores Juan N. Corpas y Roberto Serpa.

En diciembre de 1925 el Hospital fue trasladado a los denominados Molinos de la Hortúa, situado en la carrera 10a. con calle primera sur, su ubicación actual, ocupando la Maternidad el edificio del sector oriental, compartido con los servicios de Clínica Tropical y la de Organos de los sentidos. Estos servicios fueron posteriormente trasladados, quedando la totalidad del edificio para la Sección de Obstetricia, con alrededor de 200 camas.

En el año de 1930 los jefes del servicio eran los profesores Nicolás Buendía y Miguel Rueda Acosta. En ese mismo año se creó la consulta externa o prenatal cuyo primer jefe fue el Dr. Nicolás Torres Barreto y el Dr. Luis María Ferro, su ayudante.

En mayo de 1944 mediante el Acuerdo No. 14, emanado de la Junta General de Beneficiencia de Cundinamarca, de quien dependía el Hospital, se creó el Instituto Materno Infantil de Cundinamarca, con el objeto de dar protección a la mujer embarazada y al niño desde su nacimiento, tomando como base para su organización y funcionamiento el Servicio de Maternidad del Hospital San Juan de Dios, dándole autonomía administrativa, pero dependiendo económicamente de la Beneficencia de Cundinamarca. Como primer Director fue nombrado el Profesor José del Carmen Acosta Villaveces, a quien sucedió el profesor Luis María Ferro y posteriormente lo han sido José Vicente Sánchez Zapata, Carlos Martínez Sáenz, Fernando Sánchez Torres, Jesús A. Gómez Palacino, Eduardo Cáceres Alvarez, Luis Eduardo Santamaría Páez, Rodrigo Díaz Llano, Carlos Julio Pacheco Consuegra y Roberto Carrascal Pérez, su actual Director.

\footnotetext{
* Profesor Asociado. U. Nacional.

Departamento de Obstetricia y Ginecología. U. Nacional de Colombia.
}

La organización del Instituto se inició con tres servicios, como se denomina el número de camas asignadas a cada profesor titular de Clínica Obstétrica, quienes eran asesorados por un Jefe de clínica y dos internos. Los servicios eran los de los profesores José del Carmen Acosta Villaveces, Carlos Julio Mojica y Víctor Rodríguez Aponte. Al profesor Rodríguez Aponte lo sucedió el profesor Luis María Ferro; posteriormente se crearon dos nuevos servicios los de los profesores Rafael Peralta Cayón y Rafael Ramírez Merchán y además el cargo de Profesor Agregado. Hacia el año de 1955 fueron profesores agregados los doctores: Santiago Lleras, Rafael Hunda Cruz, Fernando Mera, Jaime Corral Maldonado, Eudoro Castillo Vega, Alberto Cárdenas, Carlos Roberto Silva. Jefes de clínica los doctores: Leopoldo Saavedra, Guillermo Navas Angel, Jesús Alberto Gómez Palacino, Eugenio Pérez Gómez, Mario Sáenz, Hernando Navas Angel, Jaime Ramírez Sánchez. Los internos eran denominados temporales (primer año) e internos residentes (segundo año). Todos los cargos se obtenían por concurso. El jefe de consulta externa era el Dr. Gustavo Riaño, y el jefe de pediatría el Dr. Humberto Gómez Herrera y su Jefe de Clínica el Dr. Francisco Millán; el jefe de Salacuna el Profesor Ernesto Plata Rueda, profesores auxiliares el Dr. Fidel Torres León como Urólogo, el Dr. Enrique Riveros, como Cirujano, el Dr. Guillermo Sierra, como Radiólogo y los doctores Luis Rivera y Enrique Gutiérrez Sánchez, como Anestesiólogos.

La enseñanza de pregrado de Obstetricia se hacía en dos cursos anuales, el primero se dictaba en el 5o. año de carrera y era denominado la Cátedra de Obstetricia, que era un componente esencialmente teórico. El segundo curso en 60 . año, la Clínica Obstétrica, que tenía un componente teóricopráctico y era dictada por el profesor titular. La cátedra fue dictada hasta el año de 1946 por el Profesor Hernando Acosta Sarmiento, a partir de 1947 fue dictada por los profesores: Hernando Caycedo, Rafael Peralta y Rafael Ramírez, hasta el año de 1948, cuando lo hicieron los doctores: Enrique Archila, Hernando Navas, Eugenio Pérez y Guillermo Navas.

A raíz del nombramiento del Doctor José Vicente Sánchez, como Director del Instituto (1956), siendo Jefe de la Sección de Obstetricia el Profesor Rafael Ramírez Merchán, se le imprimió un cambio radical al funcionamiento del Instituto: se suprimieron los denominados servicios y se crearon grupos docente-asistenciales. Se establecieron las residencias en Obstetricia en la categoría de residente junior (primer año), senior (2o. año), auxiliar (3o. año) y residente jefe (4o. año). 
El personal certificado se escalonó de acuerdo con su preparación académica y cada grupo asistencial contaba con un instructor y profesores nombrados por la Facultad de Medicina. Con esa modalidad egresaron los primeros especialistas en Obstetricia, desgraciadamente sin ningún reconocimiento legal, ni académico en esa época.

La Ginecología aparece como especialidad en 1903, derivada de la denominada Patología Externa y de la Clínica Quirúrgica, en lo que hoy corresponde a la Cirugía General, gracias a los buenos oficios del profesor Rafael Ucrós Durán, quien regentara la cátedra por más de 7 lustros en calidad de Profesor Titular, distinción que ostentaba desde 1904. Posteriormente lo hicieron los profesores titulares Carlos Tirado Macías (1937), Daniel Brigard (1948), y Arturo Aparicio Jaramillo (1948).

La sección de Ginecología estuvo ubicada en el 3er. piso occidental del Hospital San Juan de Dios, existiendo la modalidad de interno, jefe de clínica, profesor agregado y profesor titular. El Consejo Directivo de la Universidad Nacional, por medio de la Resolución No. 194 del 8 de octubre de 1957, creó lo que se denominó el Servicio Quirúrgico de Postgrado, mediante el cual se autorizó el establecimiento de cursos de postgrado en las áreas quirúrgicas a partir de enero de 1958. La Resolución 307 del 24 de octubre de 1958 creó los comités docentes de postgrado, pero fue mediante la Resolución 239 de julio de 1960, la que creó el comité de Educación Graduada, encargado de la organización, ejecución y supervisión de los cursos de postgrados en las diferentes especialidades, tanto quirúrgicas como médicas.

En la sección de Ginecología se inició entrenamiento a nivel de postgrado con duración de 4 años, siendo los primeros egresados como especialistas en Ginecología los doctores: Arturo Rodríguez Soto, Rubén Darío Guzmán, Guillermo Castro y Salomón Turbay.

A raíz de la reforma curricular de la Facultad de Medicina, de la cual fuera gestor como Decano el Dr. Raúl Paredes Manrique, en el año de 1960, los programas de educación médica de pregrado dejaron de ser anuales y se diseñó el sistema de bloques, integrando materias afines como la Obstetricia y Ginecología, para ser cursadas en un semestre con intensidad horaria de tiempo completo. Se creó el escalafón docente actual de Instructor Asistente, Instructor Asociado, Profesor Asistente, Profesor Asociado y Profesor Titular, con la modalidad laboral de medio tiempo y tiempo completo. El sistema creó incompatibilidades horarias a los docentes que trabajaban en otras entidades oficiales, razón por la cual se presentó una renuncia masiva de docentes. Se recurrió entonces a confirmar en su cargo a los pocos que no renunciaron y se llamó a lo que se denominó concurso de títulos, o sea el análisis curricular para su nombramiento.

En Obstetricia el profesor Rafael Ramírez Merchán, fue nombrado como jefe de sección y como profesores Rafael Peralta Cayón, Santiago Lleras, Jesús A. Gómez Palacino, Alvaro Velasco, Fernando Sánchez, Luis Santamaría, Hernando Rozo Pardo, Héctor Henao y Jorge Flórez. En Ginecología, jefe, el profesor Guillermo López Escobar y docentes los Dres: Hernando Amaya León, Héctor Enrique Bernal, Jorge Amorocho Carreño, Alvaro Fonnegra Miramón, Eduardo Arévalo Burgos, Alvaro Espinosa Espinosa, Eduar- do Cáceres Alvarez, Jorge Villareal Mejía y Francisco Pardo Vargas. Posteriormente ingresaron como docentes Arturo Rodríguez Soto, Rubén Darío Guzmán, Guillermo Castro Márquez, Heliodoro Naáñez Burbano, Laura Rojas Rodríguez, Armando Lozano Carrillo y Armando Muñoz González.

En el año de 1961 se integró el programa de postgrado de Obstetricia y Ginecología, en un programa común de residencia, en semestres alternos en cada sección hasta el año de 1967, en el cual la Ginecología fue trasladada al Instituto Materno Infantil, funcionándose los programas docentes de pre y postgrado, siendo sus jefes los profesores Rafael Ramírez Merchán y el profesor Guillermo López Escobar, a quien reemplazó posteriormente el profesor Alvaro Fonnegra Miramón.

En el año de 1970 mediante el acuerdo 100 del Consejo Superior Universitario elevó a la categoría de Departamento de Obstetricia y Ginecología, las 2 secciones, quedando constituidas estas a su vez en unidades docentes. El primer director fue el Profesor Rafael Ramírez Merchán y posteriormente lo han sido en su orden los profesores: Hernando Amaya León, Jesús A. Gómez Palacino, Fernando Sánchez Torres, Eduardo Cáceres Alvarez; Luis E. Santamaría Páez, Armando Muñoz González, Heliodoro Ñáñez Burbano, Alvaro Velasco Chiriboga, Antonio Lomanto Morán, José D. Castillo y Luis E. Santamaría el actual Director.

El Acuerdo No. 32 del Consejo Superior Universitario de fecha 6 de mayo de 1987 en una reorganización interna de los departamentos suspendió las dos unidades docentes quedando para fines administrativos como solo Departamento. En el año de 1973 el Consejo Superior Universitario, mediante el acuerdo 33 dio aprobación a los programas presentados por los diferentes departamentos. En 1981 se solicitó una reestructuración de los mismos. El Departamento de Obstetricia y Ginecología dirigido por el Dr. Luis Armando Muñoz, presentó un programa con un concepto académico de la especialidad, con diseño de objetivos, contenido académico programático por niveles de enseñanza en unidades docentes, siguiendo una metodología y estrategia basada en el programa básico para la enseñanza de la Obstetricia y Ginecología que se sigue en los Departamentos de la especialidad en los Estados Unidos de América, que fue y sigue siendo modelo de programa académico. Inicialmente el título de especialista fue expedido por la Asociación Colombiana de Facultades de Medicina (ASCOFAME), debiendo los egresados someterse a un examen aprobatorio tanto teórico como práctico. Posteriormente a raíz de dos movimientos huelguísticos de los residentes se logró abolir el examen y que fuese la Universidad Nacional por medio de la Facultad de Medicina, quien acreditara los títulos, por disposición del Consejo Académico.

En febrero de 1963 terminó el primer especialista integrado en Ginecobstetricia, con 4 años de Residencia el Dr. Rafael Gómez García (fallecido).

Hacia los años 70 el Instituto Materno Infantil y el Hospital San Juan de Dios tuvieron que soportar la inmensa carga asistencial de una ciudad como Bogotá, que crecía en forma vertiginosa, sin los recursos presupuestales adecuados, lo cual precipitó una crisis en el centro hospitalario que dio origen a la creación de la Fundación San Juan de Dios, en el año de 1979, con lo cual se pretendió solucionar el problema administrativo. 
La Fundación tampoco funcionó y el Ministerio de Salud tuvo que intervenirla, situación que se conserva hasta la fecha.

El edificio donde funcionaba el Instituto Materno Infantil por el desgaste del tiempo llegó a un estado deplorable que obligó al Ministerio de Salud a emprender su remodelación a finales del año 1979, siendo necesario trasladar los servicios al 8o. piso del Hospital San Juan de Dios, en donde funcionó hasta el 24 de febrero de 1984, cuando fue reinagurada su planta actual.

Actualmente el Instituto Materno Infantil es un hospital universitario de tercer nivel, en donde se atiende el alto riesgo de la zona cuatro de Bogotá, pero en la práctica de referencia de todo Bogotá, Cundinamarca y muchos Departamentos aledaños.

Gracias a la remodelación cuenta con las siguientes áreas:

\section{Consulta externa de Obstetricia-Ginecología y Pediatría}

\section{Obstetricia:}
a) Prenatal-general
b) Toxemias
c) Sépticas
d) Alto riesgo
e) Diabetes
f) Esterilidad e infertilidad
g) Planificación familiar

\section{Ginecología:}
a) Ginecología general
b) Oncología
c) Incontinencia urinaria de esfuerzo
d) Endocrinología
e) Clínica de dolor
f) Valoración pre-anestésica
g) Clínica de Climaterio que incluye además Psicología, Terapia Física, Nutrición y Trabajo Social.

\section{Consulta de Admisión - Servicio 24 horas}
a) Trabajo de parto
b) Urgencias obstétricas
c) Urgencias ginecológicas

\section{Pediatría:}
a) Seguimiento de lactante-general
b) Lactante de alto riesgo
c) Cirugía general
d) Ortopedia
e) Cirugía plástica
f) Cardiología
g) Urología
h) Genética
i) Programa madre canguro
j) Urgencias pediátricas

\section{Terapias:}
a) Ocupacional
b) De lenguaje

c) Respiratoria

6. Psicología:
a) Ginecológica
b) Obstétrica
c) Pediátrica

\section{Area intrahospitalaria:}

1. Partos:
a) Sala de trabajo de parto
b) Sala de partos
c) Sala de legrados
d) Observación-recuperación

\section{Cirugía:}
a) Dos salas de ginecología
b) Sala de cesáreas
c) Sala de cirugía pediátrica
d) Sala de sépticas

\section{Ginecología-pacientes ginecológicas-quirúrgicas}

4. Toxemias

5. Sépticas

6. Alto riesgo

7. Cesáreas

8. Puérperas

\section{Legrados}

10. Servicio de Anestesiología
a) Valoración preanestésica
b) Analgésica y anestesia obstétrica
c) Anestesia ginecológica
d) Pediátrica
e) Sépticas
f) Legrados

11. Pediatría:
a) Sección de adaptación neonatal
b) Alto y mediano riesgo
c) Aislamiento
d) Recién nacido sano-puérperas
e) Recién nacido sano-cesáreas
f) Cirugía
g) Cardiología
h) Unidad de cuidado intensivo infectado
i) Unidad de cuidado intermedio no infectado
j) Lactario
k) Pediatría ambulatoria
l) Urgencias

12. Imágenes diagnósticas:
a) Radiología
b) Ecografía

13. Laboratorio:
a) General
b) Especializado endocrino

14. Anatomía patológica:
a) Citología 
b) Histopatológica

c) Morgue (autopsias)

15. Nutrición

16 Enfermería

\section{Personal docente}

Departamento de Obstetricia y Ginecología:

1. Luis Eduardo Santamaría P., Director del Departamento, Profesor Asociado.

2. Jaime Gallego Arbeláez, Coordinador del Departamento.

3. Miguel E. Aragón S., Profesor Asistente.

4. Edith Angel Muller, Instructora Asociada.

5. Juan Manuel Acuña A., Profesor Asistente.

6. Alejandro Bautista Charry., Instructor Asociado.

7. Rodrigo Calero Ossa., Instructor Asociado.

8. José David Castillo B., Profesor Asociado.

9. Rodrigo Díaz Llano., Profesor Asociado.

10. Hernando Gaitán Duarte., Profesor Asistente.

11. Pío Iván Gómez Sánchez., Profesor Asistente.

12. Antonio Lomanto Morán., Profesor Asociado.

13. Luis Vicente López Avila., Profesor Asociado.

14. Manuel Esteban Mercado Pedroza., Profesor Asistente.

15. William Onatra Herrera., Profesor Asistente.

16. Heliodoro Ñáñez Burbano., Profesor Asociado.

17. Carlos Julio Pacheco Consuegra., Profesor Asistente.

18. María Teresa Peralta Abella., Profesor Asociada.

19. Ariel Iván Ruiz Parra., Profesor Asistente.

20. Ruth Sánchez de Estrada. Profesor Asistente.

\section{Pediatria:}

1. Edgar Rey Sanabria

2. Héctor Ulloque

3. Santiago Currea

4. Roberto Carrascal Pérez

5. Gabriel Longie

6. Gabriel Díaz

7. Jorge Hernández

8. Jesús A. Niño

\section{Patología:}

1. Ricardo Alvarado Pantoja

2. Manuel Bustamante B.

\section{Anestesia:}

1. José María Lozada

\section{Imágenes diagnósticas:}

1. Luis Fernando Novoa

\section{Laboratorio:}

1. Santiago Buendía

\section{Médicos especialistas:}

a) Obstetricia - 25 adjuntos

b) Pediatría - 8 adjuntos

c) Anestesia - 19 adjuntos

A raíz de la organización del plan hospitalario nacional y la implementación de la atención médica por niveles, el Instituto Materno Infantil fue calificado como hospital de tercer nivel (Universitario), para atención de pacientes de alto riesgo obstétrico y ginecológico.

Son innumerables los trabajos científicos que en las diversas áreas se han llevado a cabo, pero es necesario resaltar: 1) en 1973, el Premio Nacional de Medicina Federico Lleras Acosta, fue concedido a los Doctores Jesús Alberto Gómez Palacino, Fernando Sánchez y Hugo Guevara Jaramillo, por su trabajo sobre «Toxemia gravídica»; 2) El mismo premio fue otorgado al Dr. Alvaro Velasco Chiriboga, por el diseño de las espátulas que llevan su apellido, instrumento que entre nosotros ha sustituido el fórceps obstétrico y 3) el Programa Canguro, creado por el Servicio de Pediatría del Instituto, bajo la dirección del Dr. Edgar Rey Sanabria e implementado por el Dr. Héctor Martínez Gómez, para el manejo ambulatorio del niño prematuro sano, mereció de la Organización Mundial de la Salud en 1991 le concediera la distinción Sasawaka Health Prize; 4) Mención Honorífica «Federico Lleras Acosta» al trabajo sobre «Diagnóstico de Sexo in Utero» al Dr. William Onatra H., 1972.

También es necesario resaltar que fue el Departamento de Obstetricia y Ginecología pionero en la implantación de la ecografía, como método diagnóstico en Colombia, gracias a la colaboración desinteresada del Dr. Luis Carlos Jiménez, efectuando 5 simposios internacionales sobre el tema.

En el presente año 1993 hay dos hechos que le imprimen nuevo impulso al Departamento: en primer lugar la aprobación por parte de la Universidad Nacional de un Programa de Postgrado en Neonatología, gracias a las instancias del Profesor Santiago Currea y en el segundo término la creación de la Unidad de Biología Reproductiva, de la cual han sido gestores los doctores: Juan Manuel Acuña y Ariel Iván Ruiz.

Como es fácil deducir el Instituto Materno Infantil y el Departamento han crecido en forma extraordinaria, de manera que se puede afirmar que hoy por hoy constituye en el primer Centro de Atención Integral de la Mujer, desde el aspecto prenatal, pediátrico, adolescente, edad reproductiva y climaterio, pero en especial, en el aspecto perinatal, en donde el binomio madre e hijo, como manifestación perfecta de lo que representa la esencia misma de la vida, tiene un santuario, en donde además se rinde culto a la ciencia, a la docencia, a la asistencia con verdadero apostolado, sacrificio y amor. 


\section{EGRESADOS}

En Obstetricia:

Gonzalo Beltrán García

Hugo Guevara Jaramillo

Luis Torrado Núñez

Delfín Valderrama

Jaime Díaz Garzón

Miguel Hurtado Peralta

Roberto Páez Vargas

Luis Sánchez Rodríguez

José Gabriel Acuña

Alfonso Durán Durán

Nelson Varón Correal

Ricardo Marceles

Eduardo Hidrobo

En Ginecología:

1960:

Arturo Rodríguez Soto

Rubén Darío Guzmán

Guillermo Castro Márquez

Salomón Turbay

Integrados: Obstetricia y Ginecología: 1963:

Rafael Gómez García

1964:

Luis Alba Palacios

Graciela Arboleda de Jaime

Uriel Acero Rodríguez

Fernando Handzer (E.U.)

1965:

Carlos Alfonso Barbosa Borbón t

Laureano Marín

Heliodoro Ñáñez Burbano

Germán Riaño Gambo

Carlos Sarria Hoyos

1966:

Adolfo Bernal

Antonio Lomanto

Laura Rojas Rodríguez

Germán Velasco Landázuri

1967:

Jorge Corzo Carrillo

Mario Concha Potes

José J. Granados Villamizar

Luis Vicente López Avila

Armando Carrillo Lozano

Aurelino Moyano Torres

Eduardo Rueda Angarita

Alvaro Fernando Pérez Muñoz

1968:

Fidel Anzola Fajardo

Clara Alvarez López

José David Castillo

Bernardo Barrios Montealegre

Melba Martínez de Uribe

Benjamín Herrera Méndez

(Tres años)

1969:

Alba Nelly Cardozo

Luis Armando Muñoz González (fallecido)†

Alvaro Torres Medina
Gustavo Urrego Landazábal

Ricardo Zarama Burbano

1970:

Germán U. Marín Ocampo

Julio C. Hernández Vega

Iván Alirio González Ortiz

José Ignacio Delgado Ortiz

Orlando Peinado Quintero

Adolfo Martínez Sánchez

Guillermina Ortiz de Ortiz

Leyla A. de Morales

1971:

Fernando Contreras Warnier

Fernando Prieto Acosta

Luis Felipe Milanés Padilla

1972:

Jaime E. Ruiz Acevedo

Jaime E. Fernández Rodríguez

Oscar Gómez León

Rafael Lombana Merlano

Emperatriz Reyes Navarro

1973:

Clara E. Zambrano de Rojas

Alfredo Segura Sánchez

Carlos Eduardo Villamizar Martín

Yana D. de Delgado

William Onatra Herrera

1974:

José V. Salamanca Zea

Reinaldo Aguas Yepes

Marcelino Muñoz Arrieta

1975:

Enrique Aaraon Rojas

Jaime Cabrera Mutis

Gustavo Márquez Mariño

1976:

Alfredo Ariza Machado

Edgar Cabal Quiceno

Roger Castillo Figueroa

Alberto Moreno Rojas

Alfredo Támara De La Ossa

1977:

Manuel Lagos Galindo

Leonardo Arzuza Estrada

Carlos Eugenio García Alvarez

Armando Moreno Gáfaro

José del Carmen Romero

1978:

Marco Antonio Hernández Romero

Nancy Helena Lafaurie Rodríguez

Carlos Alfonso Olaya Urueña

Luis Ernesto Pérez Agudelo

Luis Alberto Pérez Rincón

1980:

Consuelo Arteaga Díaz

Jairo Alberto Barragán Espinosa (fallecido)

Orlando Forero Cárdenas (fallecido)

Guillermo González González

Alvaro Jiménez Becerra 
Miguel Angel Perdomo Ramírez

Fabio Reyes Mahecha

Hernando Rodríguez Nivia

Saúl Santoyo Téllez

1982:

Arturo Franco D'Laytz

Manuel Esteban Mercado Pedroza

Carlos Julio Pacheco Consuegra

María Nelly Rangel de Gómez

Herman Redondo Gómez

Alvaro Salazar Galindo

Víctor Alfonso Toro Díaz

Luis José Velandia Espinosa

1983:

Miguel Eduardo Aragón Sánchez

Hilma Barriga Garzón

Epifanio Becerra Corredor

Hugo Rafael Benito Martínez

Víctor Manuel Rodríguez M.

1985:

Rodrigo A. Calero Ossa

Leonor Fonnegra Jiménez

Guillermo García Ojeda

Aleyda Guzmán Salazar

Fernando Pardo Castro

1986:

Carlos Alberto Cortés Vargas

Hernando Guillermo Gaitán Duarte

Jaime Gallego Arbeláez

Fernando José Portilla Pinzón

Ariel Iván Ruiz Parra

Jacinto Sánchez Angarita

Julio César Villamil Maya

1987 .

Cecilia Avila Castro

María Mercedes Castillo Jaramillo

Felipe De La Cruz Luna Toro

Buenaventura Morales López

Rodrigo Encisar Moreno Vejarano

David Ortiz Valero

Guillermo Augusto Rojas Avellaneda

Edgard Manuel Sastre Cifuentes

1988:

Ricardo Alberto Amaya Ch.

Jesús Alberto Echeverría Baquero

Pío Iván Gómez Sánchez

Julio R. Madrid Linares
Reinaldo Niño Alba

Martha Libia Ocampo Zuluaga

Janeth Pulido Gacharná

Jaime Celiano Rojas Cárdenas

1989:

Blanca del Rosario Fuquen Jiménez

Carmen Doris Garzón Olivares

Andrés Gordillo Lombana

Héctor Fabio Lizcano Supelano

Alfredo A. Lomanto Castañeda

Hernando Ortiz Almeida

Farid Roberto Rodrigo Monroy

Nidia Astrid Sánchez García

1990:

Catalina Acevedo Schoenhbohm

Margarita Arbeláez Pinto

Lucio Guerra Galue

Darío Rafael Guzmán P.

Pedro Pablo Jiménez $M$.

José Agustín León L.

Pedro Ignacio Rozo Reina

1991:

Francisco Javier Cuevas C.

Carlos Alberto Díaz R.

Luis Daniel Forero Q.

Miguel Alberto Rodríguez G.

Claudia Rodríguez U.

Nicolás Ruiz Cortés

Manuel Horacio Sánchez J.

María Yolanda Torrente V.

1992:

Jairo Amaya Guío

Jairo Arenas Gamboa

Gustavo A. Caycedo D.

Jorge Cuenca Urbina

Armando Díaz Quimbayo

Robinson Fernández Mercado

Susy Manotas Char

Edith Angel Muller

1993:

Saúl Pérez Echeverry

Meyer Serrano Riaño

Alejandro Bautista Charry

Julio Roberto Moreno Díaz.

Gustavo Medina Hurtado

María Consuelo Barragán Torres

Fernando Martínez Martínez

Carlos Miguel Mateus Lugo

\section{BIBLIOGRAFIA}

1. Historia de Colombia. Fundación Misión Colombia. Villegas Editores, Agosto 1988.

e. Śnchez TF. Historia de la ginecobstetricia en Colombia. Impreso por Giro Eaıtores Ltda., 1973 1a. Edición.
3. Archivo del Departamento de Ginecología y Obstetricia.

4. Archivo de la Oficina de Postgrado. Facultad de Medicina de la Universidad Nacional. 